\title{
Différentes composantes de la radioactivité naturelle et les fluctuations selon le lieu*
}

\author{
A. BOUVILLE** \\ (Manuscrit reçu le 21 décembre 1984)
}

\begin{abstract}
RESUME
Ce document donne une vue générale de la radioactivité naturelle et des expositions humaines associées. Toute forme de vie sur terre est inévitablement liée aux expositions à la radioactivité naturelle, qui a deux origines : l'espace extra-terrestre (rayonnements cosmiques) ; la formation de la terre (les substances radioactives présentes dans la croûte terrestre). Ces deux sources de radioactivité naturelle irradient le corps humain de l'extérieur mais également de l'intérieur par suite de l'inhalation et de l'ingestion des produits radioactifs. En vivant dans un environnement naturel, l'homme est exposé à toutes ces sources. En moyenne, l'exposition à la radioactivité naturelle est plus importante que les expositions aux sources artificielles.

Les sources extra-terrestres et terrestres seront considérées successivement. Une part plus importante sera consacrée aux expositions internes tout en considérant également les expositions externes. Les expositions seront exprimées en terme d'équivalent de dose effectif, qui est une quantité proportionnelle au risque de radioactivité. Une grande partie des informations est issue du dernier rapport du Comité scientifique des Nations-Unies pour l'étude des effets des rayonnements ionisants (UNSCEAR), qui revoit régulièrement les informations relatives aux sources et aux effets des rayonnements ionisants.
\end{abstract}

\section{ABSTRACT}

The purpose of this paper is to give an overview of the natural radiation environment and of the human exposures from natural radiation sources. Any form of life on earth is unavoidably associated with exposure to natural radiation. There are two kinds of sources of natural radiation: sources in the extraterrestrial environment (i.e. cosmic rays); terrestrial sources (i.e. the radioactive substances in the earth's crust). Both types of sources irradiate the human body from outside but also from inside as naturally occuring nuclides are taken up into the body through normal physiological pathways. When living in a natural environment,

* Communication présentée lors des journées organisées par la Société française de radioprotection sur "les données actuelles sur la radioactivité naturelle", MonteCarlo, 5-7 novembre 1984 .

** Commissariat à l'énergie atomique, Institut de protection et de sûreté nucléaire, IPSN/DPr, BP 6, 92260 Fontenay-aux-Roses. 


\section{A. BOUVILLE}

man is exposed to all these sources. On the average, the exposure from natural radiation is much greater than the exposures from artificial sources.

The extraterrestrial sources and the terrestrial sources will be considered in succession. Some emphasis will be placed on the internal exposures but external exposures will also be discussed. The exposures will be expressed in terms of effective dose equivalent, which is a quantity assumed to be proportional to the radiation risk. Most of the information will be taken from the latest UNSCEAR [1] report, which periodically reviews the information on the sources and effects of ionizing radiation.

Toute forme de vie sur terre subit une exposition aux sources naturelles de rayonnements ionisants, qui relèvent de deux origines très distinctes : l'espace extra-terrestre, source du rayonnement cosmique, la formation de la terre, qui donna naissance aux radionucléides primordiaux.

Ces deux types de sources contribuent à la fois à l'exposition externe et à l'exposition interne de l'homme (fig. 1). Certaines sources entraînent des expositions qui sont, dans une grande mesure, indépendantes du lieu où l'on se trouve ou du mode de vie tandis que d'autres sont extrêmement sensibles à ces facteurs. La variabilité des niveaux d'exposition sera discutée. Pour faciliter les comparaisons entre les diverses sources, les expositions seront exprimées en termes d'équivalent de dose effectif, quantité que l'on suppose généralement être proportionnelle au risque sanitaire. La plus grande partie des informations données dans ce document est extraite du rapport UNSCEAR de 1982 [1].

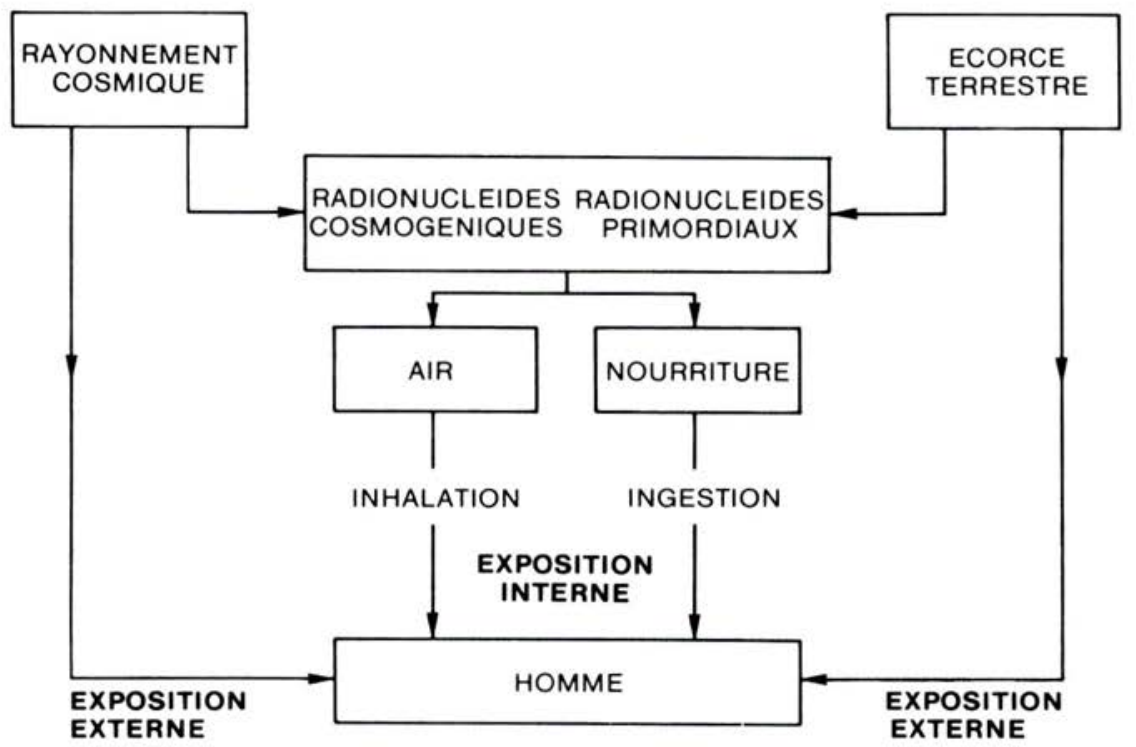

Fig. 1. - Expositions aux sources naturelles de rayonnements ionisants. 


\section{RAYONNEMENT COSMIQUE}

Le rayonnement cosmique primaire prend naissance dans l'espace extra-terrestre. II est essentiellement dû à une pluie de particules électriquement chargées (protons, hélions, électrons) en provenance du soleil, de notre galaxie et d'autres galaxies. Ces particules chargées, d'une part, sont déviées par le champ magnétique terrestre, d'autre part, interagissent avec les atomes et noyaux présents dans l'atmosphère, entraînant une dégradation du rayonnement cosmique primaire, qui devient rayonnement cosmique secondaire, et la production d'un grand nombre de radionucléides dits cosmogéniques, tels que ${ }^{3} \mathrm{H}$ et ${ }^{14} \mathrm{C}$.

\subsection{Exposition externe}

L'exposition externe au rayonnement cosmique est presque entièrement due au rayonnement cosmique secondaire. Au niveau du sol, la majeure partie de l'exposition provient des muons, qui sont des particules très pénétrantes, de sorte que les débits d'exposition sont à peu près les mêmes à l'intérieur et à l'extérieur des bâtiments. Cependant, un certain degré de protection est apporté par les bâtiments importants ; une réduction de l'exposition d'environ $15 \%$ correspondrait à une épaisseur de béton de $20 \mathrm{~cm}$, qui filtre ainsi une partie de la composante douce du rayonnement cosmique. En moyenne, l'équivalent de dose effectif annuel dû à l'exposition externe au rayonnement cosmique est d'environ $300 \mu \mathrm{Sv}$ au niveau de la mer, ce qui constitue approximativement $15 \%$ de l'exposition totale aux sources naturelles de rayonnements ionisants. La variation de l'exposition externe au rayonnement cosmique varie peu en fonction de la latitude tandis que la variation selon l'altitude est beaucoup plus importante (doublement tous les 1500 m environ) (fig. 2). Les populations de Téhéran et de Mexico reçoivent ainsi une exposition au rayonnement cosmique plus de deux fois supérieure à celle de Paris, Londres ou Amsterdam. En avion commercial, le débit d'exposition est environ 100 fois plus élevé qu'au niveau de la mer.

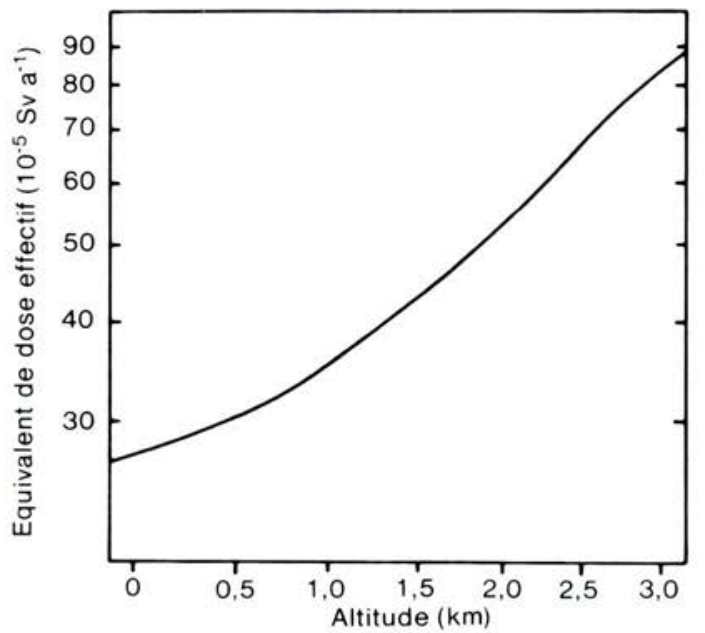

Fig. 2. - Variation avec l'altitude de l'équivalent de dose dû au rayonnement cosmique (particules chargées) à la latitude gèomagnétique de $50 \circ \mathrm{N}$. 


\subsection{Exposition interne}

Les radionucléides cosmogéniques sont principalement produits dans la haute atmosphère par l'interaction du rayonnement cosmique primaire avec l'azote, l'oxygène et l'argon de l'air. Ils parviennent au niveau du sol au bout d'un temps compris entre quelques semaines et quelques années après leur production, ce qui conduit à une bonne homogénéisation sur l'ensemble du globe. Les radionucléides cosmogéniques présentent peu d'importance sur le plan de l'exposition; seuls ${ }^{3} \mathrm{H},{ }^{7} \mathrm{Be},{ }^{27} \mathrm{Na}$ et ${ }^{14} \mathrm{C}$ méritent d'être signalés, la contribution la plus importante revenant au ${ }^{14} \mathrm{C}$ (tableau I). Comme le montrent les études dendrochronologiques ${ }^{(1)}$, le carbone 14 a été produit à un taux relativement constant au moins depuis quelques milliers d'années ; comme le carbone 14 a une période radioactive suffisamment longue pour qu'un équilibre ait èté atteint dans tous les compartiments de la biosphère, l'équivalent de dose effectif annuel dû au carbone 14 est à peu près le même pour tous les individus. II est évalué à $12 \mu \mathrm{Sv}$ par an, ce qui représente moins de $1 \%$ du total.

TABLEAU I

Radionucléides cosmogéniques

\begin{tabular}{|l|c|c|c|c|}
\hline & ${ }^{3} \mathrm{H}$ & ${ }^{7} \mathrm{Be}$ & ${ }^{14} \mathrm{C}$ & ${ }^{22} \mathrm{Na}$ \\
\cline { 2 - 5 } & 12 & 0,15 & 5700 & 2,6 \\
$\begin{array}{l}\text { Période (années) } \\
\begin{array}{l}\text { Taux de production } \\
\left.\text { (atomes } \mathrm{m}^{-2} \mathrm{~s}^{-1}\right)\end{array}\end{array}$ & 2500 & 800 & 20000 & 0,9 \\
$\begin{array}{l}\text { Dose annuelle } \\
(\mu \mathrm{S} v)\end{array}$ & 0,01 & 3 & 12 & 0,2 \\
\hline
\end{tabular}

\subsection{Discussion}

Les expositions au rayonnement cosmique sont relativement faibles et présentent un degré de variabilité peu important. Sur le plan pratique, le seul facteur à prendre en compte est l'altitude, qui joue un rôle déterminant dans l'évaluation de la composante externe de l'équivalent de dose effectif. Le débit d'exposition externe double en première approximation tous les $1500 \mathrm{~m}$, ce qui conduit à des expositions relativement importantes pour les populations vivant à haute altitude ou voyageant fréquemment en avion.

\section{RAYONNEMENT TERRESTRE}

Le rayonnement terrestre, ou tellurique, est dû aux radionucléides primordiaux qui ont été créés lors de la formation de la terre et dont la période radioactive est assez longue pour qu'ils soient encore présents dans tous les constituants de l'environnement (sols, roches, air, eaux, produits végétaux et animaux). Comme le montre le tableau II, on distingue ceux dont la désintégration donne naissance à un produit stable (comme ${ }^{40} \mathrm{~K}$ ou ${ }^{87} \mathrm{Rb}$ ) et ceux qui appartiennent à une chaîne de filiation

(1) Dendrochronologie : datation et modifications de l'environnement par l'étude des cernes de croissance des arbres. 
radioactive (principalement, les chaînes de ${ }^{238} \mathrm{U}$ et de ${ }^{232} \mathrm{Th}$ ). Les concentrations de ${ }^{40} \mathrm{~K},{ }^{238} \mathrm{U}$ et ${ }^{232} \mathrm{Th}$ dans l'écorce terrestre varient d'un facteur 100 et plus, avec des concentrations moyennes d'environ $25 \mathrm{~Bq} \mathrm{~kg}^{-1}$ pour ${ }^{238} \mathrm{U}$ et ${ }^{232} \mathrm{Th}$ et de $200 \mathrm{~Bq} \mathrm{~kg}{ }^{-1}$ pour ${ }^{40} \mathrm{~K}$.

TABLEAU ॥

Radionucléides primordiaux

\begin{tabular}{|l|c|c|}
\hline \multirow{2}{*}{${ }^{40} \mathrm{~K}$} & Période (années) & Produit de filiation \\
\cline { 2 - 3 }${ }^{87} \mathrm{Rb}$ & $1,310^{9}$ & Stable \\
${ }^{238} \mathrm{U}$ & $4,810^{10}$ & Stable \\
${ }^{232} \mathrm{Th}$ & $4,510^{9}$ & Radioactif \\
${ }^{235} \mathrm{U}$ & $1,410^{10}$ & Radioactif \\
& $7,110^{8}$ & Radioactif \\
\hline
\end{tabular}

\subsection{Exposition externe}

L'exposition externe au rayonnement terrestre a pour cause principale l'activité contenue dans les roches, le sol ou la chaussée lorsqu'on se trouve à l'extérieur des bâtiments ; à l'intérieur, ce sont les matériaux de construction qui constituent la cause essentielle.

En ce qui concerne l'exposition à l'extérieur des bâtiments, les résultats des diverses campagnes de mesures effectuées à travers le monde montrent que le débit de dose absorbée moyen dans l'air est d'environ $510^{-8} \mathrm{~Gy} \mathrm{~h}^{-1}$, ce qui correspond à un équivalent de dose effectif annuel d'approximativement $60 \mu \mathrm{Sv}$ si l'on admet que $20 \%$ du temps est passé dehors. En gros, la variabilité autour du débit de dose moyen est d'un facteur deux dans les deux sens, comme le montre la figure 3 , mais avec des exceptions notables. Par exemple, dans l'état indien du Kerala, l'équivalent de dose effectif annuel moyen reçu par les 20000 personnes les plus exposées est proche de $10000 \mu \mathrm{Sv}$. Dans plusieurs régions du Brésil, on a relevé des doses à l'air qui correspondent à des équivalents de dose effectifs annuels compris entre $3000 \mu \mathrm{Sv}$ et $20000 \mu \mathrm{Sv}$.

A l'intérieur des bâtiments, l'exposition externe est essentiellement due à l'activité présente dans les matériaux de construction. Le débit de dose moyen dans l'air est, en moyenne, $20 \%$ plus élevé à l'intérieur qu'à l'extérieur des bâtiments si les matériaux de construction sont d'origine locale. Cependant, un rapport bien plus important peut être observé si les concentrations en radionucléides naturels des matériaux de construction sont plus élevées que dans les sols environnants; c'est parfois le cas pour des matériaux d'origine naturelle tels que le granit, ou pour des matériaux incorporant des déchets industriels comme le phosphogypse ou les cendres volantes. L'équivalent de dose effectif annuel dans des maisons construites en granit peut atteindre 3000 à $4000 \mu \mathrm{Sv}$. 


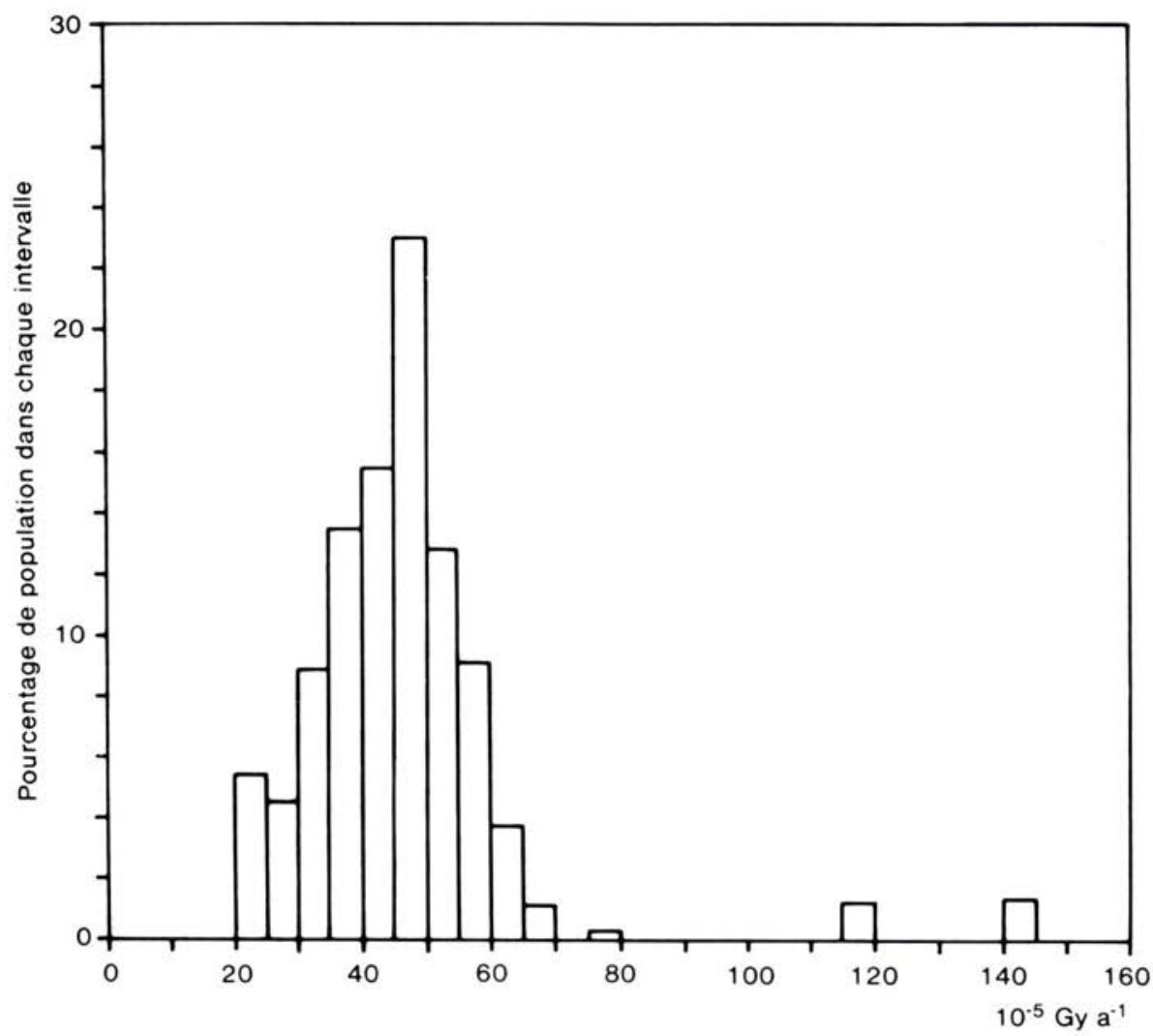

Fig. 3. - Distribution pondérée sur la population de l'exposition au rayonnement terrestre à l'extérieur des bâtiments.

\subsection{Exposition interne}

Les deux principales voies d'atteinte de l'homme par exposition interne sont l'inhalation et l'ingestion. Etant donné la variabilité des concentrations en radionucléides naturels dans l'atmosphère et dans le régime alimentaire, les niveaux d'incorporation varient d'un endroit à l'autre. Pour un lieu donné, des différences dans la composition du régime alimentaire impliquent également des différences dans les activités incorporées.

Parmi les radionucléides primordiaux, ${ }^{40} \mathrm{~K}$, et peut être aussi ${ }^{87} \mathrm{Rb}$, doivent être mis à part, car les doses résultant de leur incorporation sont sensiblement les mêmes pour tous les individus. Le potassium est un élément essentiel qui est sous contrôle homéostatique dans le corps humain, ce qui signifie que sa concentration dans les organes et tissus est indépendante de la quantité incorporée. L'équivalent de dose effectif annuel dû à l'incorporation de ${ }^{40} \mathrm{~K}$ est d'environ $180 \mu \mathrm{Sv}$, ce qui représente près de $10 \%$ du total. Cependant, de faibles variations se produisent en raison de différences dans la morbidité et la morphologie des individus considérés (fig. 4). 
L'uranium 238 (fig. 5) et le thorium 232 (fig. 6) sont chacun à la tête d'une chaîne radioactive de plus de 10 nucléides qui peuvent se trouver séparés les uns des autres par des moyens physiques, chimiques, ou biologiques. Tous les nucléides de ces deux chaînes sont des isotopes d'éléments solides, à l'exception de ${ }^{222} R n$ et de ${ }^{220} R n$, qui sont gazeux. L'inhalation et l'ingestion sont les deux voies d'atteinte à considérer.

\subsubsection{Inhalation}

L'exposition par inhalation peut résulter de deux mécanismes : remise en suspension dans l'atmosphère de particules du sol ; émanation de ${ }^{222} \mathrm{Rn}$ et de ${ }^{220} \mathrm{Rn}$ à partir du sol, des matériaux de construction et de l'eau.

La remise en suspension découle de la perturbation physique du sol sous l'effet du vent ou du passage de véhicules. On admet généralement que la remise en suspension est le mécanisme principal expliquant la présence dans l'air des précurseurs de ${ }^{222} R n$ et de ${ }^{220} R n$. L'équivalent de dose effectif annuel moyen résultant de l'inhalation des précurseurs du ${ }^{222} R$ et du ${ }^{220} \mathrm{Rn}$ est très faible ; il s'élève à environ $20 \mu \mathrm{Sv}$ soit $1 \%$ du total.

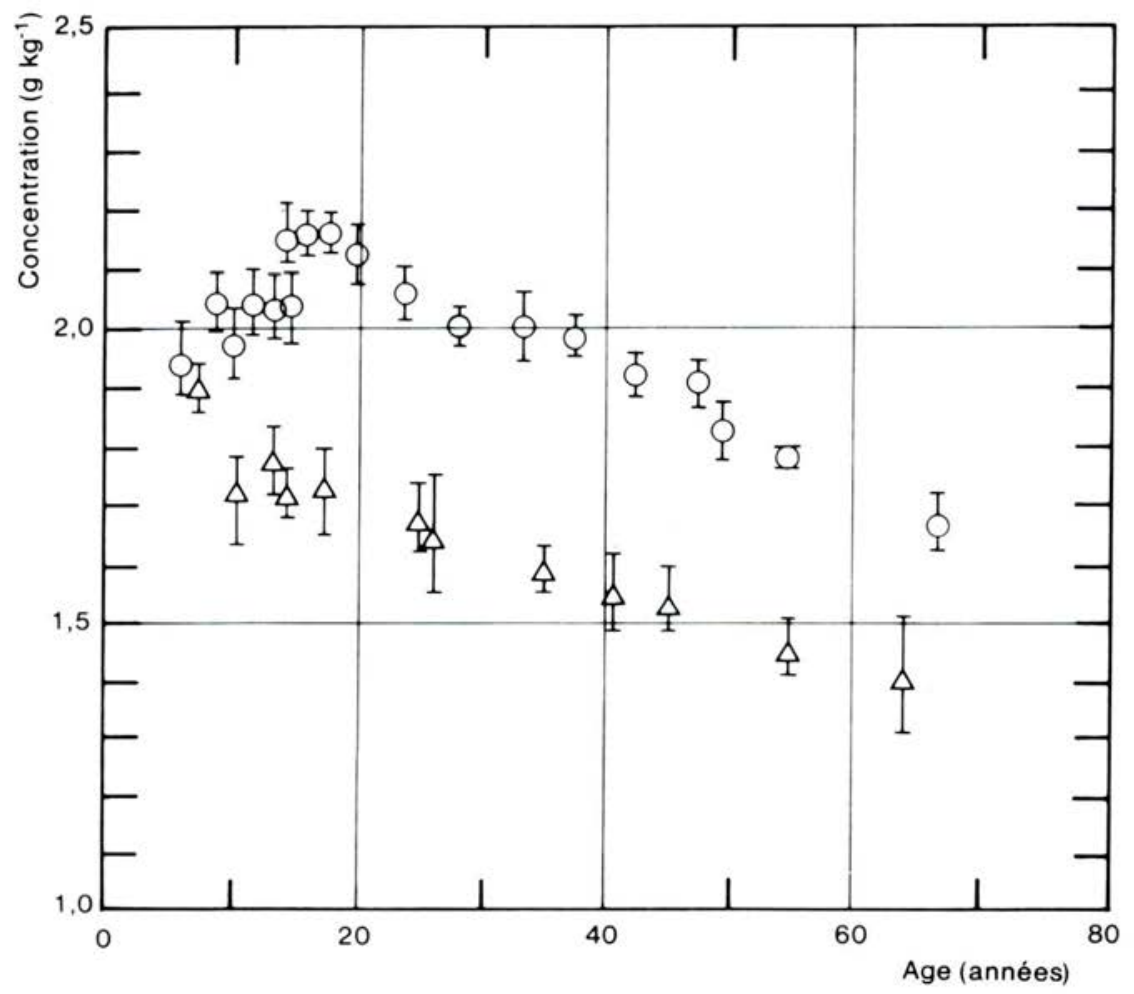

Fig. 4. - Variation de la concentration en potassium dans le corps humain en fonction de l'âge; les hommes sont représentés par des cercles et les femmes par des triangles. 


\section{A. BOUVILLE}

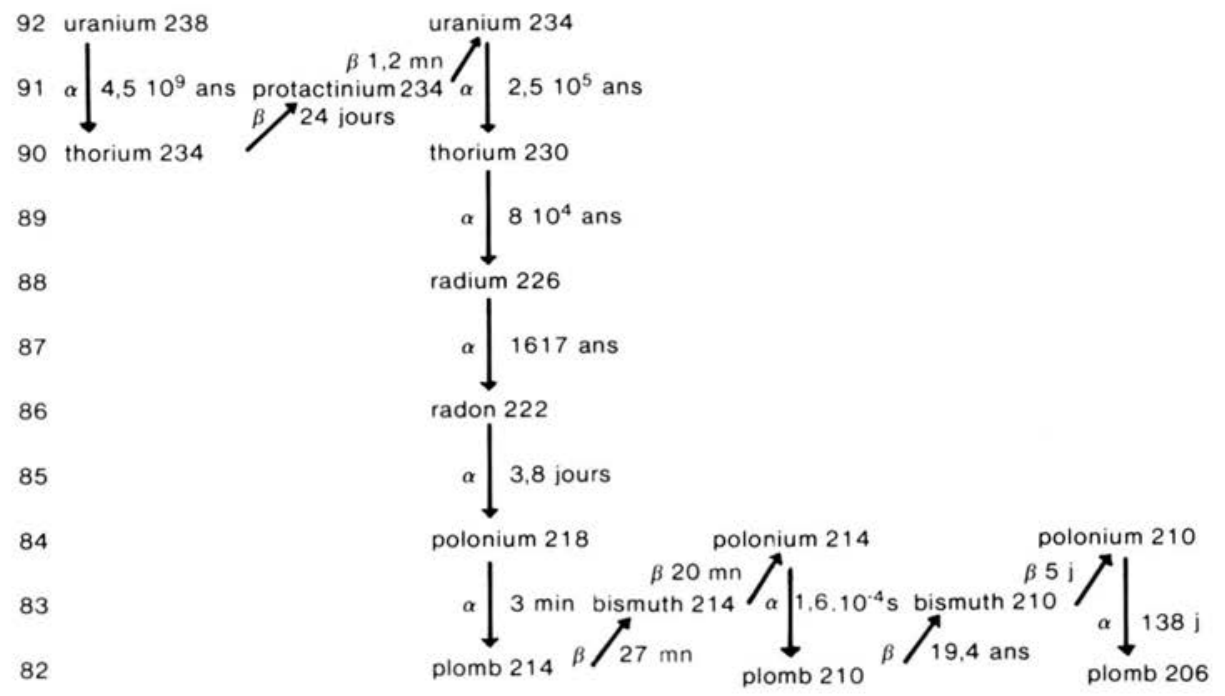

Fig. 5. - Famille radioactive de l'uranium 238

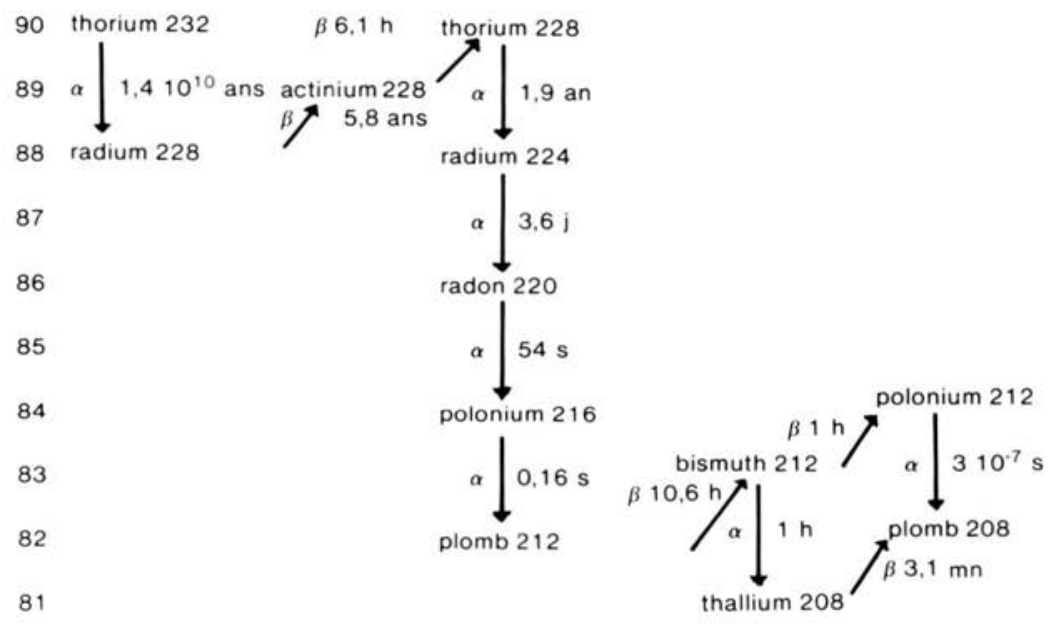

Fig. 6. Famille radioactive du thorium 232 
L'émanation de radon $\left({ }^{222} \mathrm{Rn}\right)$ et de thoron $\left({ }^{220} \mathrm{Rn}\right)$ à partir du sol, des matériaux de construction et de l'eau est un mécanisme beaucoup plus important pour ces gaz. Après leur émanation, le radon et le thoron se dispersent dans l'air où ils donnent naissance par désintégration à une série de produits de filiation radioactifs. L'inhalation de ces radionucléides conduit, en moyenne, à un équivalent de dose effectif annuel d'environ $1000 \mu$ Sv dans les zones tempérées, ce qui représente la moitié du total.

\section{- Exposition aux descendants du radon à l'extérieur des bâtiments}

Les concentrations de radon, de thoron et de leurs produits de filiation dans l'air au niveau du sol et à l'extérieur des bâtiments dépendent essentiellement de l'endroit où l'on se trouve et des conditions météorologiques. En ce qui concerne le radon et ses produits de filiation, les concentrations les plus élevées sont observées sur les continents lors des inversions de température (jusqu'à $100 \mathrm{~Bq} \mathrm{~m} \mathrm{~m}^{\cdot 3}$ ) tandis que les concentrations les plus faibles sont trouvées sur les océans et les îles lors des périodes de forte turbulence atmosphérique (minimum d'environ $0,1 \quad \mathrm{~Bq} \mathrm{~m}^{-3}$ ). Les concentrations sont également faibles dans les régions recouvertes d'une épaisse couche de neige ou de glace, telles que les zones polaires. La concentration moyenne des produits de filiation du radon au-dessus des continents est d'environ $2 \mathrm{~Bq} \mathrm{~m}^{3}$, ce qui correspond à un équivalent de dose effectif annuel d'environ $60 \mu \mathrm{Sv}$, soit $3 \%$ du total. En ce qui concerne les produits de filiation du thoron, on trouve une concentration moyenne de $0,2 \mathrm{~Bq} \mathrm{~m}^{-3}$ et un équivalent de dose effectif annuel moyen de $20 \mu \mathrm{Sv}$.

\section{- Exposition aux descendants du radon à l'intérieur de bâtiments}

L'exposition à l'intérieur des bâtiments est due principalement aux produits de filiation à vie courte du radon. Leur concentration dépend de facteurs multiples, tels que les concentrations en ${ }^{226} \mathrm{Ra}$ dans le sol et les matériaux de construction, l'épaisseur et la porosité des murs et du plancher, le revêtement des murs, le taux de ventilation de l'air,... De plus, l'utilisation d'eau ou de gaz naturel présentant des concentrations élevées de radon peut apporter une contribution significative.

Les concentrations des produits de filiation de radon à l'intérieur des habitations sont de l'ordre de $15 \mathrm{~Bq} \mathrm{~m}^{-3}$ dans les zones tempérées, ce qui conduit à un équivalent de dose effectif annuel moyen d'environ $900 \mu \mathrm{Sv}$, si l'on suppose que les gens passent $80 \%$ de leur temps à l'intérieur des habitations. Etant donné que les concentrations dans les zones tropicales sont probablement plus faibles que dans les zones tempérées, la moyenne de l'équivalent de dose effectif annuel à l'échelle du globe est estimée à environ $800 \mu \mathrm{Sv}$. Les variations d'un bâtiment à l'autre sont très importantes : les concentrations mesurées s'étagent entre 1 et $10000 \mathrm{~Bq} \mathrm{~m}^{3}$. II est clair que les doses dues à l'inhalation des produits de filiation du radon sont extrêmement variables et peuvent, dans certains cas, être très élevées.

Pour ce qui est des zones tempérées, on estime que la concentration moyenne des descendants du thoron à l'intérieur des bâtiments est de $0,7 \mathrm{~Bq}^{-3}$, ce qui entraîne un équivalent de dose effectif annuel approchant $200 \mu$ Sv. 


\subsubsection{Ingestion}

Les doses par ingestion sont fonction de la concentration des radionucléides primordiaux dans la boisson et les aliments ainsi que du taux de consommation de ces aliments. La concentration des radionucléides primordiaux dans l'eau de boisson est, en règle générale, très faible, bien que des niveaux importants soient parfois observés dans les eaux souterraines. Pour le radium 226, par exemple, l'eau de boisson tirée des rivières présente habituellement des concentrations inférieures à $10 \mathrm{~Bq} \mathrm{~m}^{-3}$, ce qui correspond à des équivalents de dose effectifs annuels d'environ $2 \mu \mathrm{Sv}$ pour une consommation quotidienne d'un litre. Cependant, dans les eaux de boisson extraites des nappes phréatiques, on a observé aux Etats-Unis des concentrations comprises entre 40 et $3000 \mathrm{~Bq} \mathrm{~m}^{-3}$. Dans le cas des eaux minérales, des concentrations élevées sont souvent obtenues : c'est ainsi qu'en France, des concentrations approchant $3000 \mathrm{~Bq} \mathrm{~m}^{-3}$ ont été mesurées dans des eaux de table.

Comme pour l'eau, la concentration en radionucléides primordiaux des produits alimentaires est généralement faible : les incorporations quotidienne de ${ }^{226} \mathrm{Ra},{ }^{210} \mathrm{~Pb}$ et ${ }^{210} \mathrm{Po}$ s'étagent habituellement entre 0,01 et $0,1 \mathrm{~Bq}$. II existe pourtant des exceptions notables. La mieux connue est celle des dizaines de milliers de personnes dans les zones arctiques et subarctiques de l'hémisphère nord qui se nourrissent en abondance de viande de renne ou de caribou. Cette viande présente des concentrations en ${ }^{210} \mathrm{Po}$ très élevées, car ces animaux se nourrissent pendant I'hiver de lichens qui accumulent ${ }^{210} \mathrm{~Pb}$ et ${ }^{210} \mathrm{Po}$. Des incorporations quotidiennes typiques sont de $0,4 \mathrm{~Bq}$ pour ${ }^{210} \mathrm{~Pb}$ et de $4 \mathrm{~Bq}$ pour ${ }^{210} \mathrm{Po}$, ce qui conduit à des équivalents de dose effectifs annuels d'environ $1000 \mu \mathrm{Sv}$.

\section{CONCLUSION}

Le tableau III récapitule la contribution de diverses sources naturelles à l'exposition des populations vivant dans les zones "normales" de radioactivité naturelle. L'exposition interne constitue à peu près le double de l'exposition externe. Parmi les radionucléides contribuant le plus à l'exposition interne, les produits de filiation du radon ressortent nettement car ils sont responsables d'environ $60 \%$ de la dose. Viennent ensuite, par ordre décroissant d'importance, ${ }^{40} \mathrm{~K}(13 \%)$, les produits à vie courte du thoron $(13 \%)$ et ${ }^{210} \mathrm{~Pb}-{ }^{210} \mathrm{Po}(8 \%)$. En ce qui concerne l'exposition externe, la dose due au rayonnement cosmique est légèrement inférieure à celle due au rayonnement terrestre.

II ne faut toutefois pas perdre de vue que les doses dues à la radioactivité naturelle varient beaucoup d'un individu à l'autre et qu'en particulier des doses relativement importantes sont reçues par divers groupes de population, tels que ceux vivant en haute altitude, dans des zones de radioactivité naturelle élevées, ou dans certaines habitations.

\section{REFERENCES}

[1] United Nations Scientific committee on the effects of atomic radiation (UNSCEAR). Ionizing radiation: levels and biological effects, report to the general assembly. New York: United Nations, 1982. 
TABLEAU III

Equivalent de dose effectif annuel moyen dû aux sources naturelles de rayonnements ionisants $(\mu \mathrm{Sv})$

\begin{tabular}{|c|c|c|c|}
\hline \multirow[b]{3}{*}{ Rayonnement cosmique } & \multicolumn{3}{|c|}{$\mu \mathrm{Sv} \mathrm{a}^{-1}$} \\
\hline & $\begin{array}{c}\text { Exposition } \\
\text { externe }\end{array}$ & $\begin{array}{l}\text { Exposition } \\
\text { interne }\end{array}$ & Total \\
\hline & & & \\
\hline Rayonnement & 300 & - & 300 \\
\hline Radionucléides cosmogéniques & - & 15 & 15 \\
\hline Rayonnement terrestre & & & \\
\hline${ }^{40} \mathrm{~K}$ & 120 & 180 & 300 \\
\hline Chaine de ${ }^{238} \mathrm{U}$ & & & \\
\hline${ }^{238} \mathrm{U} \longrightarrow{ }^{226} \mathrm{Ra}$ & & 25 & \\
\hline${ }^{222} \mathrm{Rn} \longrightarrow{ }^{214} \mathrm{Po}$ & 90 & 800 & 1045 \\
\hline${ }^{210} \mathrm{~Pb} \longrightarrow{ }^{210} \mathrm{Po}$ & & 130 & \\
\hline Chaîne de ${ }^{232} T h$ & & & \\
\hline${ }^{232} \mathrm{Th} \longrightarrow{ }^{224} \mathrm{Ra}$ & 140 & 20 & 330 \\
\hline${ }^{220} \mathrm{Rn} \longrightarrow{ }^{208} \mathrm{TI}$ & 140 & 170 & 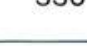 \\
\hline Total arrondi & 650 & 1350 & 2000 \\
\hline
\end{tabular}

